\title{
STRUCTURAL AND MODULAR ARCHITECTURES FOR THE DESIGN OF SMART MECHATRONIC AND CYBER-MIXMECHATRONIC SYSTEMS USED IN LABORATORIES AND IN THE INDUSTRY
}

\author{
Gheorghe I. Gheorghe1, Iulian Ilie ${ }^{2}$ \\ 1,2 National Institute of Research and Development in Mechatronics \\ and Measurement Technique, Bucharest \\ geocefin@yahoo.com
}

\begin{abstract}
This scientific paper shows the structural and modular architectures for the design of smart mechatronic and cyber-mixmechatronic systems used in laboratories and in the industry.
\end{abstract}

Keywords: Structural and Modular Architecture, Smart Mechatronic Systems; CyberMixmechatronic Systems.

\section{Introduction}

The design of structural and modular architectures is based on the paradigm of smart mechatronic and cyber-mixmecatronice systems with functions of measuring and integrated control, handling, etc. automated in automated and cybernetized industrial processes.

In the synthesis of the scientific work is presented the design of informatic models for different industrial applications is, economic and societal ones.

\section{Experimental Models}

(2.1) The design of the informatic model - cyber-mixmechatronic robot system with remote monitoring and remote control (fig. 1)

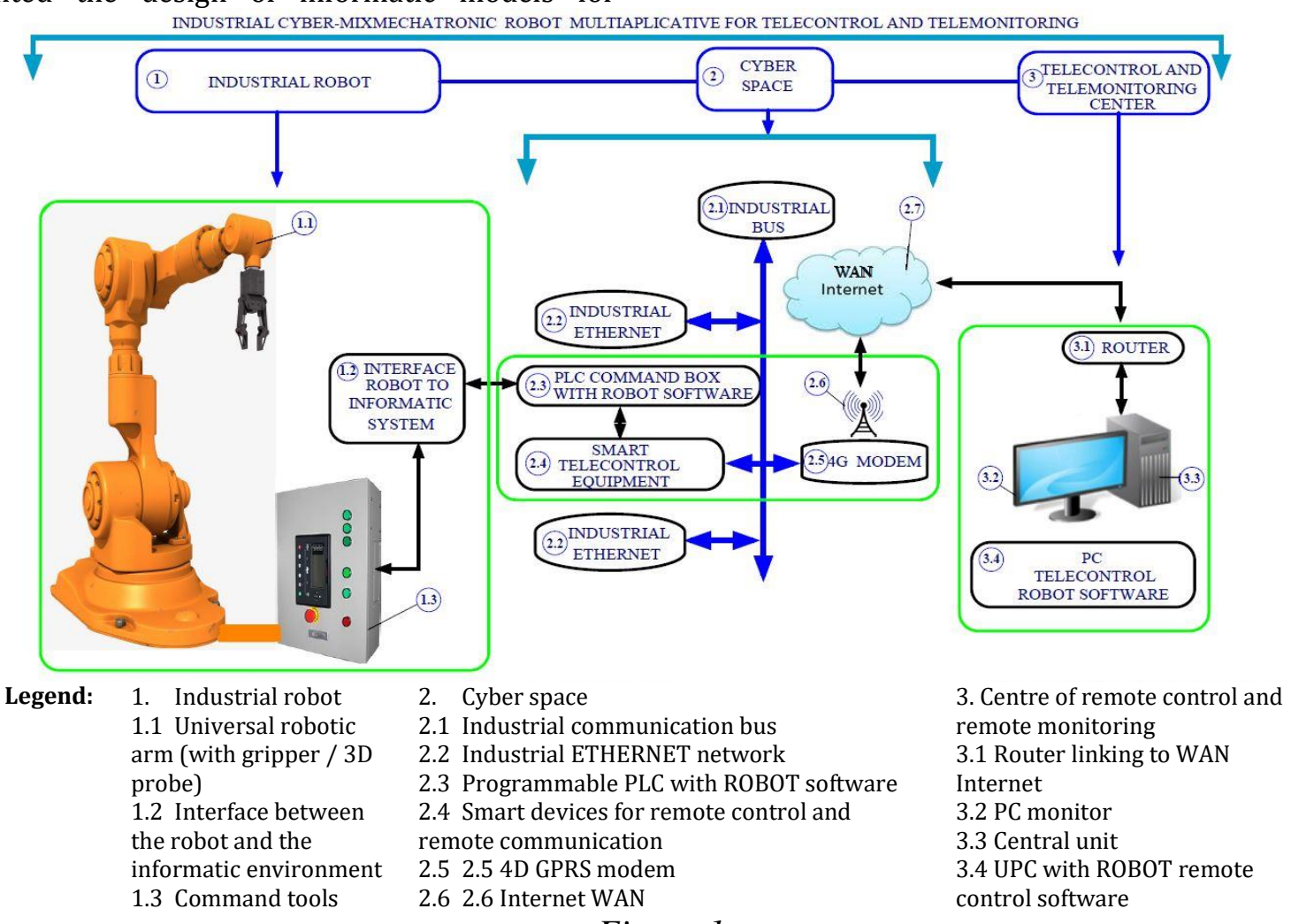

Figure 1 
- The smart industrial robot (Figure 1) has a complex structure consisting of:

$>$ The robot itself with a smart gripper arm and a 3D probe

$>$ Robot- Computing environment interface;

$>$ Control unit with PC.

- The robot through the interface makes the connection to the PLC and communications systems.

- Cyberspace, that is a complex structure consisting of:

$>$ Industrial communication bus;

$>$ Industrial Ethernet network;

$>$ PLC programmed with robot software;

$>$ Smart remote control and remote communications equipment;

4G GPRS MODEM;

$>$ Antenna

$>$ WAN Internet.

The cyberspace makes connection with the Robot via the robot interface and with the remote control centre via the centre router.

- The remote control and maintenance centre has a structure formed of:

$>$ WAN Internet connection router;

$>$ PC monitor;

$>$ PC central unit;

$>$ UPC with robot remote control software.

$>$ The link of the centre with the cyberspace is made via the router.

As an architectural ensemble, the information flow of the "cyber0mixmechatronic system with remote monitoring and remote control" derives from the non-electrical "input variables", which are converted into electrical quantities, then amplified, split and displaying, resulting in "the output variables" in the form of product, service and technology", a result that can be taken into account as such, or may be controlled, monitored or configured from the distance via the interconnections of cyberspace and of the remote control and remote monitoring centre.

The remote control and remote monitoring cyber-mixmecatronic robot system is used in any industrial process or company based on its constructive and functional integration, in smart manufacturing processes, either its own or as control and remote maintenance manufacture system, assembly lines or lines of quality assurance.

The actual robot is architecture structurally and functionally depending on process requirements where it is integrated, and especially on the gripper's architecture, so that the robot becomes a "smart control equipment" or a "technological and/or industrial services equipment".

In this regard, gripper can be interchangeable, so it can have integrated the "3D probe" for entering into contact with the surfaces of the workpiece / part that needs to be controlled or the integration of the gripper with "gripping, application, sales, transportation or handling" devices in processes related to technological applications.

\section{(2.2) The design of the informatic model - technological cyber-mixmechatronic remote monitoring and remote control mixmecatronic instrument system (Fig. 2)}

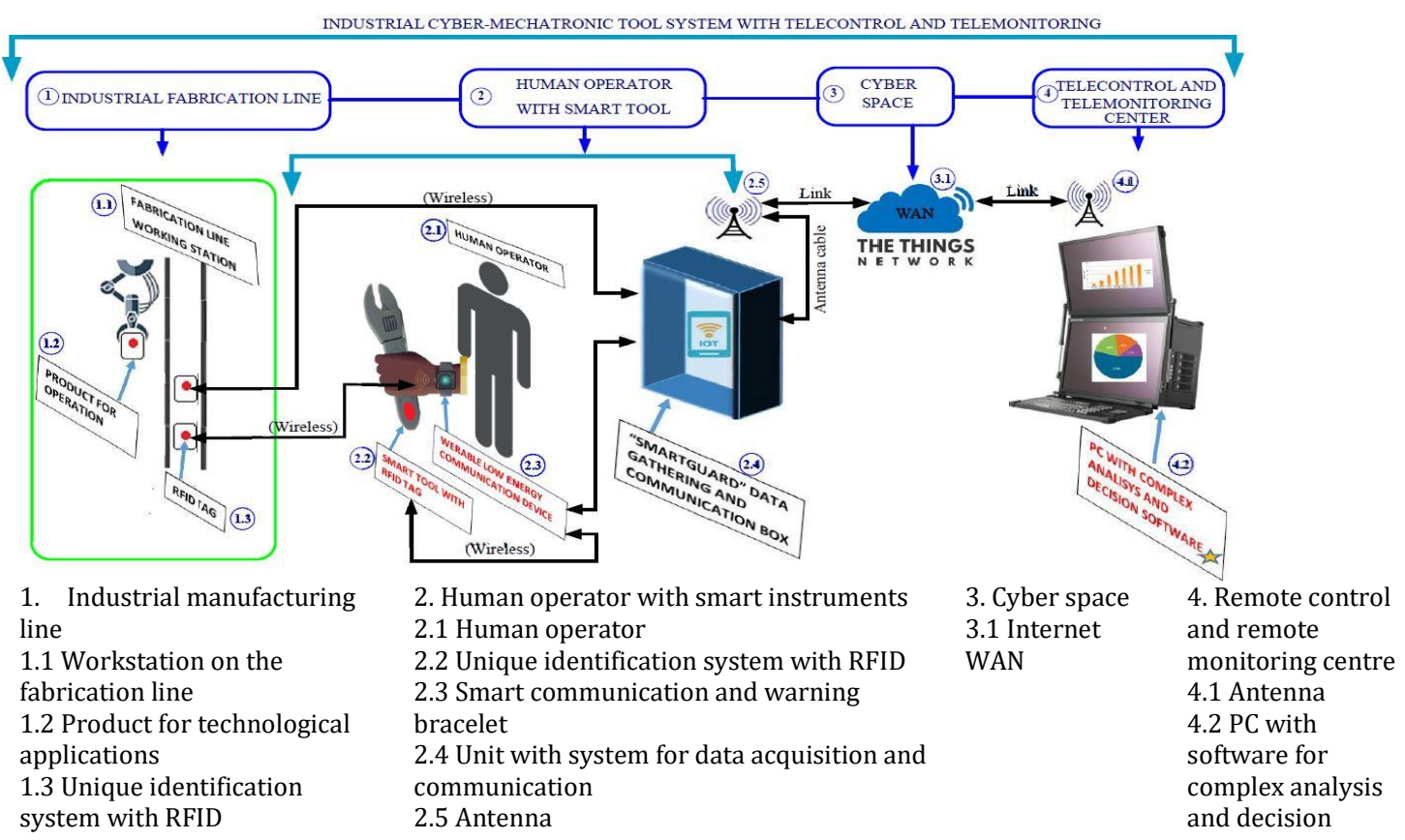

Figure 2 
The design of the technological cybermixmechatronic remote monitoring and remote control mixmecatronic technological instrument system (Figure 2) comprises a multi-complex structure consisting of:

(1) Industrial robot manufacturing line;

(2) Human operator with integrated smart tool;

(3) Cyberspace;

(4) Remote control and remote monitoring centre.

- The industrial robot manufacturing line (Figure 2) has a technological structure specific to the cybernetization, comprising the following:

$>$ Technological work station in the production line;

Related product and its specific technological operations;

$>$ Unique identification system with RFID.

The link between the robotized industrial fabrication line and the human operator is made wirelessly, for data collection and communication

- The human operator has integrated smart technology tools (Figure 2), including:

unique RFID identification tool, smart communication and warning bracelet, unit with a system of data collection and communication and antenna. The link between the human operator equipped with smart technology tools and the production line, is made wirelessly, and the cyberspace is made via the antenna and WAN Internet.

- The cyberspace is characterized by the WAN Internet (Figure 2), and linked to the human operator via the smart communication equipment (for the human operator) and is connected to the central remote control and remote monitoring centre via the link to the communication equipment of the centre.
- The remote control and remote monitoring centre (Figure 2) has the following structure: Antenna, and PC equipped with analysis software and decision-making capabilities.

\section{Remote control and telemonitoring centre}

\subsection{Antenna}

4.2 PC equipped with analysis software and decision-making capabilities

As a whole. the " technological cybermixmechatronic remote monitoring and remote control mixmecatronic instrument system" ensuring the performance of operational phases and technological activities, based on the smart technology related to the process or to manufacturing processes through the integration and connection with "human operator endowed with a smart tool" that assists and intervenes in the process of technical and technological assembling, through his or her inter-connection and decision, depending on the actual unfolding of the process and that part of a dialogue with the other virtual communication systems in order to optimize the automated processes and to ensure their quality.

Communication and information transfer required for the cybernetic automated process is done through wireless cybernetic components, facilitating the smooth integration of the human operator with the automated cybernetic systems and subsystems.

Moreover, the human operator equipped with a "smart communication and warning bracelet" can be replaced right now or in the very near future by a "humanoid robot" that can be integrated into the cybernetization of the industrial process.

(2.3) The design of the informatic model - 3D multiaplicative remote control and remote monitoring system (fig. 3)

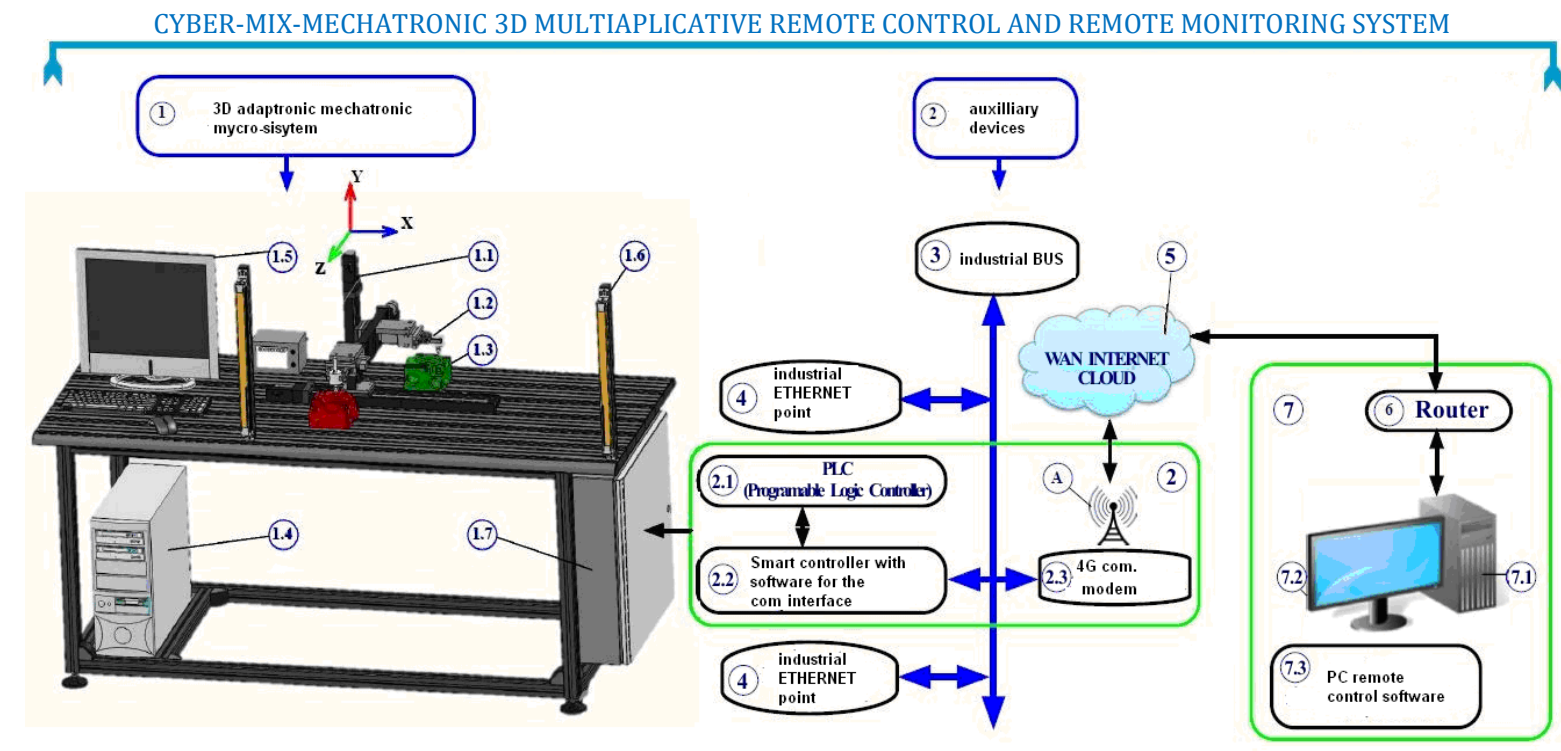




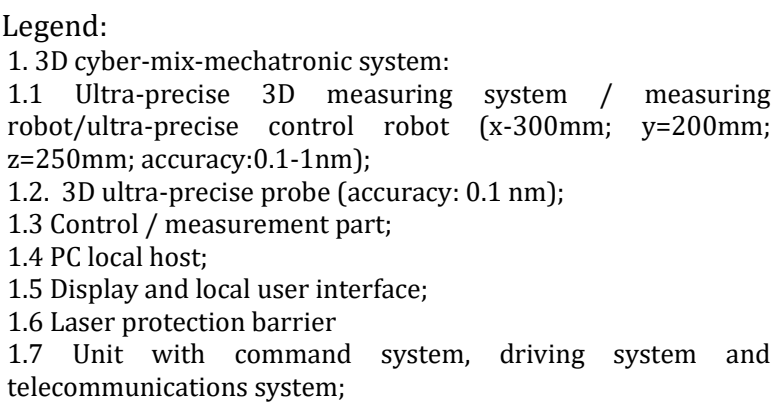

2. Auxiliary equipment:

2.1 PLC (Programmable Logic Controller);

2.2 Smart controller with software for communication interface;

$2.34 \mathrm{G}$ communication modem;

3. Industrial BUS;

4. Industrial ETHERNET point;

5. WAN INTERNET CLOUD access;

6. $802,1 \mathrm{lb} / \mathrm{g}$ router

7. Control Centre

7.2 PC Display

7.3 PC Remote monitoring software

Figure 3

This 3D cyber-mixmechatronic system can be used in following industrial environments:

- in the automotive industry for completely processed automotive parts and moulded auto parts, as the smart cyber-mixmecatronic 3D system is integrated in car production lines and car assembly, contributing thus to the cybernetization of car manufacturing;

- in the precision mechanics and mechanical industry, for miniature parts and high precision parts and in mechatronic and precision mechanics subassemblies and assemblies as the 3D cybernetized system is integrated into smart mechatronics and micromechanics assembly lines, as well as into electronics and micro-electronics and computer science and micro computer science assembly lines;

- in the aerospace industry, for small and medium-size parts, high-precision processed and moulded parts, where the 3D cyber-mechatronic system is integrated into the smart fabrication lines and assembly lines of systems and aerospace systems (e.g. microsatellites, etc.);

- in the automotive industry, for mechanical parts, machined and cast small and medium highprecision parts, where the 3D cyber-mechatronic systems are integrated into the smart control process of manufacturing;

- in the electronic and automation industry, for smart control of circuits, for controlling the precise positioning of electronic components, etc., where the 3D cyber-mechatronic systems are integrated into the smart manufacturing lines;

- in the optical industry, for smart control of mechanical and optical systems in which the cybermechatronic system is integrated into the specific production lines;

- in the environmental industry, energy industry and so on.

- The organigram structure includes:

- The 3D (adaptronic) cyber-mixmecatronic system (Fig. 3):
(1) $3 \mathrm{D} \quad(\mathrm{X}, \mathrm{Y}, \mathrm{Z})$ system with ultra-accurate movements;

(2) 3D touch probe system with submicron fidelity;

(3) Measurement part;

(4) PC central unit;

(5) Monitor unit;

(6) Safety barriers and infrared security sensor;

(7) Unit with remote control and remote communication equipment;

- The cyberspace (Fig. 3):

(1) Industrial communication bus;

(2) Industrial Ethernet network;

(3) PLC programmed by 3D software;

(4) Smart communications equipment;

(5) GPRS 4G modem;

(6) Antenna

(7) WAN Internet;

- The remote control and remote monitoring centre (Fig. 3):

(1) WAN Internet Connection Router;

(2) PC Monitor;

(3) PC central unit;

(4) UPC with 3D remote control software;

- The cyberspace of the remote control and remote monitoring centre (Fig. 3):

(1) PLC programmed with 3D software;

(2) Smart remote control equipment;

(3) GPRS 4G modem;

(4) Antenna.

(2.4) Smart multi-complex mechatronic dual 2D measurement system for smart control and smart industrial services (Fig. 4)

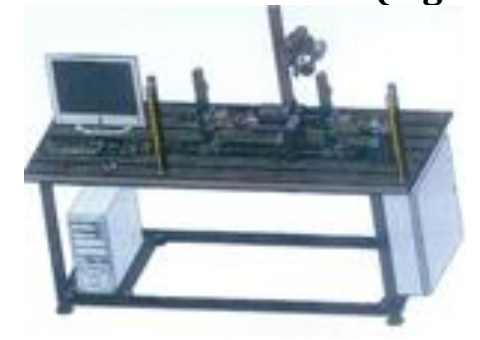

Figure 4 
This multi-complex 2D mecatronic dual system has a matrix architecture consisting of:

- subsystem of support table for "dual 2D mechatronic systems";

- (two) intelligent mechatronic 2D subsystems, each with one gripper and a 3D sensor, with adjustable position in the workspace, according to the size of the piece to be measured;

- TV camera subsystem for "the measurement" by assisting the continuous process of acquiring images and transferring them to the remote-control centre through cyberspace;

- subsystem of workspace barriers;

- PC Central Unit subsystem;

- control and coordination subsystem;

- gripping subsystem;

- 3D touch probe subsystem;

- specialized software package for process measurement, control and services.

The structural and functional design of a 3D intelligent mechatronic system can be extended and developed in complex structures and adaptive multicomplex industrial processes and industrial environments to their coverage and to the needs of quality standards of products.

Thus, we can design and build a "complex 3D mechatronic and cyber-mechatronic system", with repetition of the overall construction, their Cartesian axes in a 'tree-like' structure, covering all areas and productive fabrications of industrial and societal fabrications.

You can build such a "multi-complex systems" with $1 \mathrm{X}, 2 \mathrm{X}, 3 \mathrm{X}, 4 \mathrm{X}$, etc. repetitions, and $2 \mathrm{D}$ or $3 \mathrm{D}$ axes in order to cover all areas of intelligent manufacturing technology, ensuring quality.

\section{(2.5) Smart 3D mechatronic equipment, developed with two smart structures} (Fig. 5).

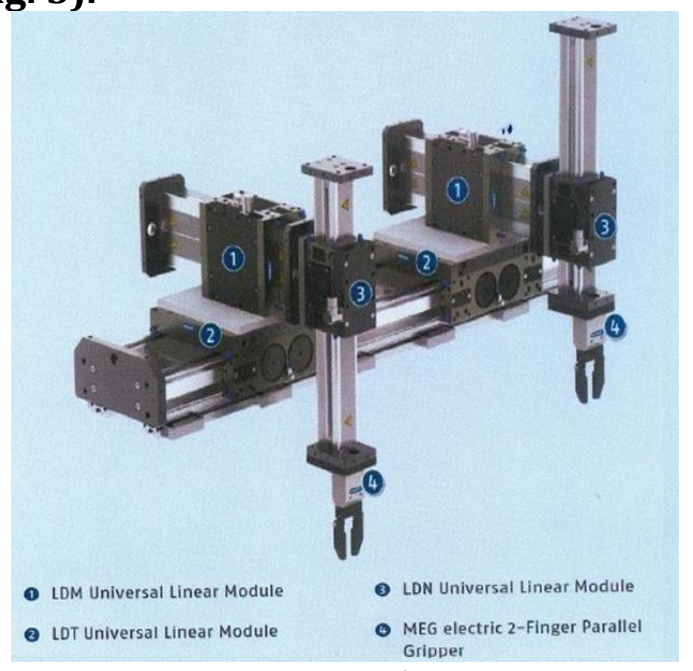

Figure 5
This cyber-mechatronic and mechatronic equipment (depicted in Figure 5) will be included in the construction assembled with virtual systems ICT in order to form a "System Overview" of complex 3D cyber-mechatronic and mechatronic equipment integrated in mechatronic intelligent control industrial processes related to manufacturing in different smart industries, in order to achieve the following:

- The control and coordination of operational phases;

- The central PC with auxiliary devices for transfers of information, processing information, setting up and managing databases and active dialogue and interconnections;

- The cyber space for connections to remote mechatronic and cyber-mechatronic systems and equipment and Internet and Intranet;

- The remote control and remote monitoring centre and the mechatronic equipment integrated in the mix-mechatronic manufacturing processes.

\section{Conclusions}

This scientific work is dedicated to «phase 2 « of «Nucleu« programme focusing on the modular design and simulation of various organizational schemes of mechatronic (adaptronic) and cybermechatronic and cyber-adaptronic systems and equipment and focus their industrial and societal applications as answers to major societal challenges.

\section{References}

[1]R. H. Bishop: The Mechatronics Handbook, The University of Texas at Austin, Austin, Texas, 2002;

[2]F. W. Bruns, H. H. Erbe, M. Faust: Engineering Future Laboratories, In: Marvel - A Leonardo da Vinci Pilot Project- Mechatronics Training in Real and Virtual Environments, Bremen, 2005

[3]B. D. Noble and J. Flinn. 2007. Wireless, selforganizing cyber-physical systems. NSF Workshop on Cyber-Physical Systems. Austin, TX.

[4]H. Gharavi, K. V. Prasad, and P. Ioannou. 2007. Scanning advanced automobile technology. Proc. IEEE, 95, 328-333.

[5]H. Zhang. Overview of wireless cyber-physical systems (WCPS). [online]. Available at http://www.cs.wayne.edu/hzhang/courses/826 0/Lectures/.

[6]Bowles, P., Tiller, M., Elmqvist H., Brück D., Mattsson S.E., Möller A., Olsson A., Otter, M.: Feasibility of Detailed Vehicle Modeling. SAE World Congress, paper 01P-321, Detroit, 2001. 